\title{
Performance monitoring of the GE1/1 Triple-GEM detectors for the CMS Muon System
}

\author{
Gabriele Milella $^{a, *}$ and Federica Simone ${ }^{a}$ on behalf of the CMS collaboration \\ ${ }^{a}$ Department of Physics, University of Bari, Bari, Italy \\ E-mail: gabriele.milella@cern.ch, federica.maria.simone@cern.ch
}

The muon system of the CMS experiment has been instrumented with a new station of triple-GEM detectors in order to ensure redundancy in the pseudo-rapidity region $1.55<|\eta|<2.18$, keeping the trigger rate at an acceptable level while not compromising the CMS physics potential in Run 3 of the LHC. The station, named GE1/1, provides two additional muon track hit measurements which will improve the muon tracking and triggering performance in combination with the existing CSC detectors. As the commissioning phase of the detector is ongoing, prompt assessment of the muon detection performance is crucial for adjusting the operating parameters of the detector and its electronics. This contribution will present a set of analysis tools developed for the detector performance monitoring based on tools common to all the CMS muon subdetectors. Validation of the analysis based on simulations will be discussed, together with preliminary results obtained from cosmic-ray events.

The Ninth Annual Conference on Large Hadron Collider Physics - LHCP2021

7-12 June 2021

Online

${ }^{*}$ Speaker 


\section{Introduction - GE1/1 station for the upgrade of the CMS forward muon system}

The CMS experiment [1] at LHC [2] is currently facing a period of major upgrades in order to cope with the High Luminosity LHC Phase (HL-LHC) requirements [3].

Several modifications of all the CMS subdetectors are therefore foreseen and, in particular, the muon system will undergo significant improvements as new detectors will be installed in the forward region. A new station of detectors, denoted GE1/1 and based on triple Gas Electron Multiplier (GEM) technology, has already been installed during the second Long Shutdown (LS2), complementing the first station of Cathode Strip Chambers (CSC), called ME1/1. Other two stations of GEM detectors, denoted GE2/1 and ME0, will be installed respectively in 2022 and 2025 [4].

The GE1/1 station covers the pseudorapidity region $1.55<|\eta|<2.18$ and it consists of 72 chambers per endcap, each covering $10^{\circ}$ in the azimuthal angle $\phi$. In the GE1/1 station a pair of triple-GEM chambers is combined to form a "superchamber", providing two measurement planes. Due to mechanical constraints, GE1/1 superchambers are divided in short and long ones for their pseudorapidity acceptance: short chambers cover the range of $1.61<|\eta|<2.18$, while long ones the range of $1.55<|\eta|<2.18$. A GE1/1 single chamber is segmented in eight sectors in $\eta$, called eta partitions (i $i$ ), where $i \eta=8$ is the closest partition to the beam line, and in 3 sectors in the $\phi$ direction for a total of 24 sectors, each containing 128 readout strips readout by the VFAT3 chip [5].

\section{GE1/1 Performance Studies}

An analysis targeting the efficiency and spatial resolution of the GE1/1 detectors has been developed based on simulated muon samples from $\mathrm{p}-\mathrm{p}$ collisions and from cosmic rays. The analysis has been tested on promptly reconstructed data collected by CMS during some commissioning runs of data taking with cosmics. It provides feedbacks on the detector performance on a short time scale (few days after data taking), thus being an important tool for the Run 3 operations.

\subsection{Detector performance in $\mathbf{p}-\mathbf{p}$ collisions environment}

The GE1/1 performance in $\mathrm{p}-\mathrm{p}$ collisions is examined with simulated $\mathrm{Z} \rightarrow \mu^{+} \mu^{-}$samples. Simulated events are generated and reconstructed to reproduce the Run 3 conditions: the setup for the simulation is therefore based on $\mathrm{p}-\mathrm{p}$ collisions at $\sqrt{s}=13 \mathrm{TeV}$, bunch crossing of $25 \mathrm{~ns}$ and with the default magnetic field value of $3.8 \mathrm{~T}$. No pile-up events are generated.

The main GE1/1 detectors parameters to be investigated are the muon detection efficiency and the spatial resolution, evaluated from the residual distribution. The starting point of the analysis workflow is the reconstruction of muon tracks in the muon system only: the points of the tracks corresponding to each subdetector are referred as reconstructed hits [6]. After applying a selection on the muon reconstruction quality selection and selecting those with $p_{T}>20 \mathrm{GeV}$, the tracks that cross both GE1/1 and ME1/1 stations are propagated from ME1/1 stations to the active surface of the GE1/1 chambers up to a point called propagated hit.

The efficiency is then defined as the ratio between the propagated hits matching with reconstructed hits within $|R \Delta \phi|<1 \mathrm{~cm}$ divided by all the propagated hits, where $R$ refers to the $R$-coordinate of the propagated hits and $\Delta \phi$ is the difference in $\phi$ position between propagated and reconstructed hits. Figure 1 shows the computed efficiency as a function of muon $p_{T}$ and $\eta$ : 

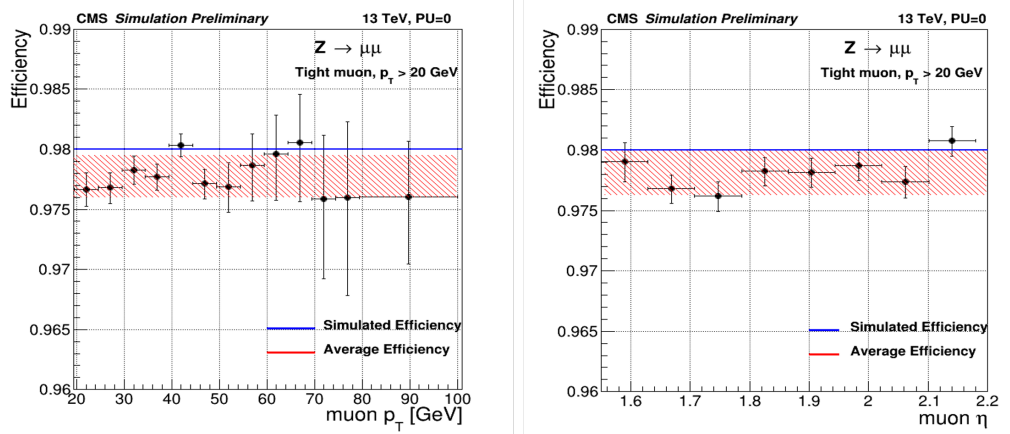

Figure 1: Detection efficiency as a function of muon $p_{T}$ (left) and $\eta$ (right). The blue curve reflects the results from test beams of $98 \%$, while the red band is the $3 \sigma$ range of statistics uncertainty around the average efficiency value. Efficiency drops are related to the simulated GE1/1 electronics noise and to large angle scattered tracks.
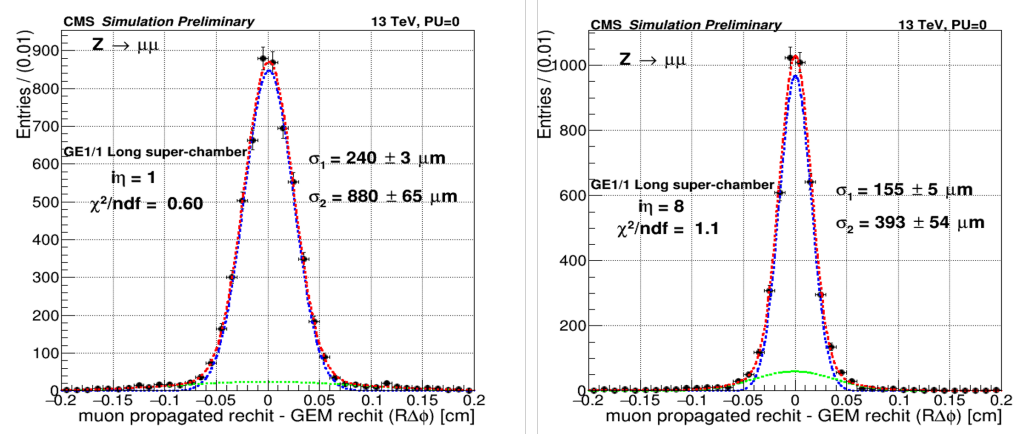

Figure 2: $R \Delta \phi$ residual distributions of the GE1/1 i $\eta$ one (left) and eight (right) of long superchambers. The distribution is fitted with two Gaussian functions and an exponential function. Red curve represents the cumulative fit while the blue and green the two Gaussian functions. The values of $\sigma_{1}, \sigma_{2}$, obtained from the fit, along with the $\chi^{2} /$ n.d.f. value are displayed in the plots.

the three $-\sigma$ value around the average computed value is compatible with the $98 \%$ efficiency value used to simulate the detector response in the sample. The residuals are computed as the distribution of the $R \Delta \phi$ values after the propagated and reconstructed hits matching. The computation is done separately for each $i \eta$ and for long and short superchambers since their readout boards have different strip pitches. Figure 2 shows an example of the $R \Delta \phi$ distribution for $i \eta$ one and eight of long superchambers. The distributions are fitted with a double Gaussian function that accounts for the core and the tails of the distribution, and with an exponential function for the background.

\subsection{Detector performance with cosmic-rays events}

By the end of 2020, the GE1/1 station has been fully integrated in the CMS muon system. First cosmics data after the installation were taken during the MWGR which is a period up to three days aimed to commission the new detectors and to monitor all the subsystems in preparation to the future physics data taking period.

During the MWGRs, the GE1/1 station has participated in the global data taking and the response of the detectors has been monitored investigating the distribution of the reconstructed hits across 
the GE1/1 station surfaces. The overall occupancy plot of the reconstructed hits is a powerful tool to spot problems arising during the data taking, such as electronic noise or DAQ issues.

As an example, in the MWGR of April 2021, 137 out of 144 GE1/1 chambers were successfully readout: due to DAQ issues, seven chambers were not powered on. Moreover, approximately $0.4 \%$ of the active VFAT readout chips experienced noise issues resulting in an higher number of reconstructed hits. Figure 3 (Left) shows the occupancy plot of reconstructed hits in the first layer of GE1/1 station of the negative endcap.

Figure 3 (Right) shows a cosmic ray muon candidate track obtained combining the reconstructed hits of the GEM (GE1/1) and CSC (ME1/1 and ME3/1) detectors collected during the MWGR of November 2020.
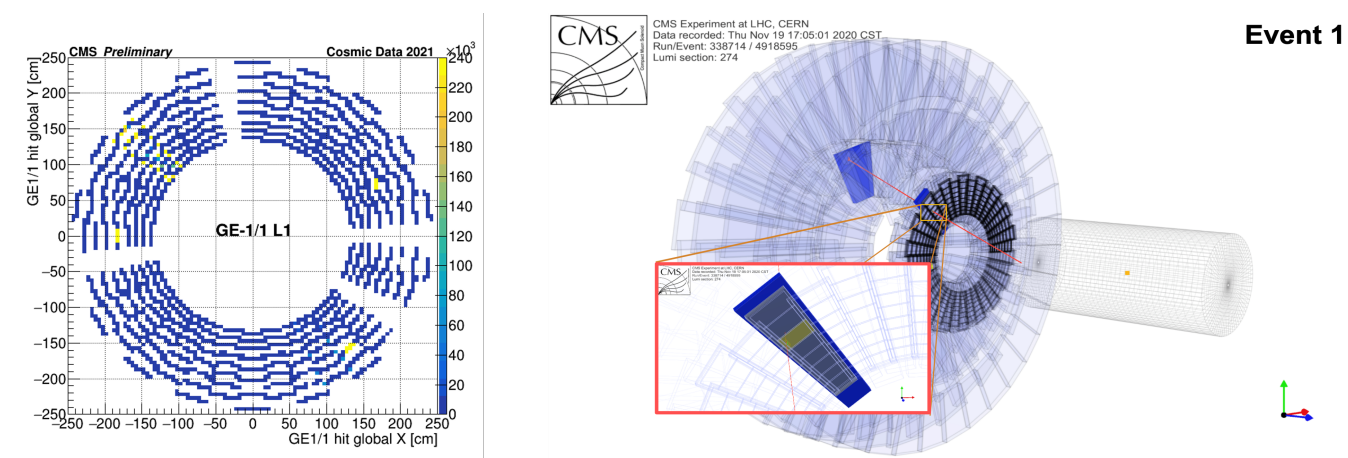

Figure 3: (Left) The GE1/1 reconstructed hits occupancy plot in the $\mathrm{x}-\mathrm{y}$ plane of the first layer in the negative endcap during the April 2021 MWGR. The blank spaces represent the chambers that were not powered on, while chambers exhibiting an higher occupancy due to electronic noise are highlighted in yellow. (Right) A $3 \mathrm{D}$ event display of a cosmic muon candidate recorded in the negative endcap during the November 2020 MWGR. The muon track (in red) releases a hit in ME3/1 and ME1/1 chambers (in blue) and in i $\eta$ six of the GE1/1 superchamber (in yellow).

\section{Conclusion}

The results presented for both simulation and data has proven the validity of the analysis workflow for retrieving and monitoring the GE1/1 detector performance: these results are the preliminary studies on GE1/1 performance after the installation. In $p-p$ collisions scenario, the computed efficiency is found to be compatible with the expected value of $98 \%$. The study of the reconstructed hits occupancy of the GE1/1 station in the MWGRs has given an indication of the issues arisen during the data taking.

The future goal is to use the workflow to extend these studies to the next long data taking runs of CRUZET (Cosmic RUn at ZEro Tesla) between July and August 2021, CRAFT (Cosmic Run At Four Tesla), scheduled for September 2021, and for the early collisions in Run 3 in 2022. 


\section{References}

[1] S. Chatrchyan et al. "The CMS Experiment at the CERN LHC". In: JINST 3 (2008), S08004. DOI: $10.1088 / 1748-0221 / 3 / 08 /$ S08004.

[2] Lyndon Evans and Philip Bryant. "LHC Machine”. In: Journal of Instrumentation 3.08 (Aug. 2008), S08001-S08001. DoI: 10 . 1088/1748-0221/3/08/s08001. uRL: https : //doi . org/10.1088/1748-0221/3/08/s08001.

[3] The HL-LHC project. URL: https : / / hilumilhc . web . cern . ch / content/hl - lhcproject.

[4] The Phase-2 Upgrade of the CMS Muon Detectors. Tech. rep. CERN-LHCC-2017-012. CMSTDR-016. This is the final version, approved by the LHCC. Geneva: CERN, 2017. URL: https://cds. cern. ch/record/2283189.

[5] P. Aspell et al. "VFAT3: A Trigger and Tracking Front-end ASIC for the Binary Readout of Gaseous and Silicon Sensors". In: 2018 IEEE Nuclear Science Symposium and Medical Imaging Conference Proceedings (NSS/MIC). 2018, pp. 1-8. DoI: 10.1109/NSSMIC . 2018. 8824655 .

[6] The CMS Collaboration. "Performance of the CMS muon detector and muon reconstruction with proton-proton collisions at $\mathrm{s}=13 \mathrm{TeV}$ ". In: Journal of Instrumentation 13.06 (2018), P06015-P06015. ISSN: 1748-0221. DOI: 10 . 1088/1748-0221/13/06/p06015. URL: http://dx.doi.org/10.1088/1748-0221/13/06/P06015. 